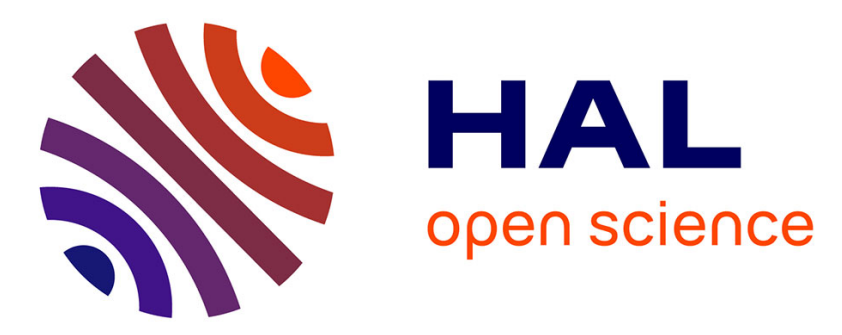

\title{
DPMS: A Swift Data Dissemination Protocol Based on Map Splitting
}

Sabri Allani, Taoufik Yeferny, Richard Chbeir, Sadok Ben Yahia

\section{To cite this version:}

Sabri Allani, Taoufik Yeferny, Richard Chbeir, Sadok Ben Yahia. DPMS: A Swift Data Dissemination Protocol Based on Map Splitting. 40th IEEE Annual Computer Software and Applications Conference, COMPSAC 2016, Atlanta, GA, USA, June 10-14, 2016, Jun 2016, Atlanta, United States. pp.817-822, 10.1109/COMPSAC.2016.100 . hal-01908072

\section{HAL Id: hal-01908072 \\ https://hal-univ-pau.archives-ouvertes.fr/hal-01908072}

Submitted on 14 Jan 2020

HAL is a multi-disciplinary open access archive for the deposit and dissemination of scientific research documents, whether they are published or not. The documents may come from teaching and research institutions in France or abroad, or from public or private research centers.
L'archive ouverte pluridisciplinaire HAL, est destinée au dépôt et à la diffusion de documents scientifiques de niveau recherche, publiés ou non, émanant des établissements d'enseignement et de recherche français ou étrangers, des laboratoires publics ou privés. 


\section{DPMS: A swift data Dissemination Protocol based on Map Splitting}

\author{
Sabri Allani \\ LIPAH-LR 11ES14, \\ University of Tunis \\ El Manar \\ 2092 Tunis, Tunisia \\ sabri.allani@gmail.com
}

\author{
Taoufik Yeferny \\ LISI LAB \\ University of Carthage \\ Tunis, Tunisia \\ yeferny.taoufik@gmail.com
}

\author{
Richard Chbeir \\ LIUPPA LAB \\ University of Pau \\ and Adour Countries \\ 64600 Anglet, France \\ richard.chbeir@univ-pau.fr
}

\author{
Sadok Ben Yahia \\ LIPAH-LR 11ES14 \\ University of Tunis \\ El Manar \\ 2092 Tunis,Tunisia \\ sadok.benyahia@fst.rnu.tn
}

\begin{abstract}
The main objective of the VANET networks is to improve road safety as well as transport efficiency through the use of communications technology and the emergence of wireless devices at low cost. Thus, the design of an efficient dissemination protocol, that informs vehicles about interesting safety and nonsafety events, is of paramount importance. The thriving challenge would be to maximize the delivery ratio by avoiding as far as possible the broadcast storm problem. A scrutiny of the literature wealthy number of approaches highlights that all of them fail to fulfill with the "sine qua non" requirements that we introduce. In this paper and to palliate this shortage, we introduce a new infrastructure-less geocast approach, called DPMS (data Dissemination Protocol based on Map Splitting). The main thrust of DPMS stands in reaching a high delivery ratio as well as a high geocast precision by sending messages only to vehicles in the Zone of Relevance (ZOR) with a minimum overhead cost. Carried out experiments showed that DPMS outperforms its competitors in terms of effectiveness and efficiency.
\end{abstract}

Index Terms - Vanet Networks, Dissemination, ZOR, GeoCast, Intelligent Transport System (ITS).

\section{INTRODUCTION}

Owe to embedded devices into modern cars, issues related to transportation, e.g., traffic congestion, road safety, driver comfort, are grasping the interest. In fact, thanks to these systems, vehicles are able to detect several types of information, e.g., an accident in the road, an empty place in a parking, bottling, obstacles, weather, road cut, to cite but a few. Interestingly enough, vehicles are also able to exchange such information using inter vehicle communications (IVCs) [1], which are based on short-range wireless technologies, to build a Vehicular Ad hoc Networks (VANET). In this respect, a core and challenging issue in vehicular networks is the design of an efficient dissemination protocol that informs vehicles about interesting safety and non-safety events. The thriving challenge would be to maximize the delivery ratio by avoiding as far as possible the broadcast storm problem. The latter is known to lead to network saturation as well as conflict issues and collision. A careful scrutiny of the pioneering vehicle-tovehicle dissemination approaches highlight that can be split into two categories : event-driven, scheduled, or non-demand approach. In the remainder, we will argue for choosing the event-driven approach, where data dissemination is carried out through broadcast or geocast techniques. Worth of mention, the main moan that can be addressed to the broadcast stands in the costly dissemination of messages to all the vehicles standing in the neighborhood. This drawback led us to naturally opt for the geocasting technique, which is the most feasible data dissemination approach for VANET's applications. The latter requires delivering information to specific regions and more especially in safety applications unlike do the broadcasting. Interestingly enough, an approach based on the goecast technique has to fulfill with the following requirements: $(i)$ determining the geocast area; (ii) delivering the message to the geocast region; and (iii) keeping the geocasted message in the network for desired delay, such that the disseminated information could reach all the arriving vehicles. Even though, the literature witnesses a wealthy number of geocast based technique for data dissemination, only few of them considered all of the three above mentioned requirements.

In this paper and to palliate the above mentioned drawbacks, we introduce a new infrastructure-less geocast approach, called DPMS (data Dissemination Protocol based on Map Splitting). The latter aims at reaching a high delivery ratio as well as a high geocast precision by sending messages only to vehicles in the Zone of Relevance (ZOR) with a minimum overhead cost. To meet these goals, DPMS heavily relies on a smart map decomposition of a complex urban or highway road grid with multiple road directions. Doing so, a high geocast precision and a low overhead value are reached.

The remainder of this paper is organized as follows: In Section II, we describe the pioneering approaches of the literature. In Section III, we thoroughly describe the main idea of our dissemination protocol. In Section IV, the simulation settings and the preliminary evaluation of the proposed DPMS protocol are then presented. Besides, a comparison between DPMS versus the surveyed other geocast protocols is also presented. The last section concludes the article and sketches issues of future work.

\section{RELATED WORKS}

In the past few years, Vehicular Ad-hoc Networks (VANETs) have gained a lot of attention owe to their promising suitability with regard to transport efficiency, safety, drivers' assistance and information/entertainment[2]. 
Therefore, various solutions for VANETs have been proposed to cope with message dissemination under different traffic conditions. In VANET networks data dissemination protocols can be categorized into two categories, namely, broadcast and geocast. The first one, consists in disseminating data to all vehicles. Whereas, geocast looks for disseminating data only to vehicles in a definite geographical region, called Zone Of Relevance (ZOR) [3]. Hence, geocast is the most feasible data dissemination approach for VANET's applications. In the remainder, we provide an overview of some works that tackled the problem of data geocasting in vehicular networks. Alsubaihi et al. [4] classify the existing approaches as Geometric, Path Sharing and Splitting-Based, Beacon-Based, Time Stable and Abiding geocast approaches.

In a geometric approach, the geographical area of dissemination is set by exploiting the geometric properties of the map [5]. For example, in Allal et al. [2], the ZOR is defined as many shapes like circle, triangle, or quadrilateral. Later, Hsu et al. [6], presented the geometric area as an aggregated distance from triangle's vertices to this region. After determining the ZOR, pure broadcasting or moderated broadcasting techniques, e.g., slotted-1 persistence, slotted-P persistence and weighted persistence, could be of use to disseminate the message to vehicles within the ZOR.

The SmartGeocast [7] approach is the most worth of cite one falling within the category Path, Sharing and Splitting-Based approaches. In the latter, two phases are included: geocasting initialization and geocasting maintenance. The former step relies on the definition of landmarks, tokens and messages using a digital map, event type and event position. The concept of path splitting is used when landmarks is split as head lighthouse (HLH) or tail lighthouse (TLH). The HLH is located at the upstream crossroad where an abnormal event occurs, whereas the TLH is located at the crossroad close to a target region. The concept of path sharing is used whenever multiple messages through multiple paths are aggregated into a single message and delivered through a single path, leading to reduced bandwidth consumption. During the maintenance phase, the vehicle that holds aggregated messages should retransmit them to other vehicles based on the concept of regional autonomy. Hence, this splitting of the area into small target areas can reduce the message redundancy and maintenance cost.

In Beacon-Based approach, the information is disseminated to ZOR periodically based on a one-hope time slot. The beaconing operation can be done before or during geocasting the message, in order to exchange several information (e.g., position, signal power, etc.) between neighboring vehicles. Ibrahim et al. [8] introduced a beacon-based approach named probabilistic Inter-Vehicular Geocast (P-IVG). The latter selects the farthest vehicles, standing within the transmission range, for further dissemination of the message. When several nodes have an equivalent distance and starts the same timer, P-IVG is based on vehicle density information.
In Time Stable Geocast, the message must be kept alive inside the specific geocast area, however, to deal with such thing a time stable geocast period. Worth of cite, Rahbar et al. and Kheawchaoom et al. [9] [10], respectively, introduced two versions of Dynamic Time-Stable Geocast protocol (DTSG). The main focus was to guarantee the message delivery with a low cost, such that the protocol should dynamically update the waiting stable time. The first version of DTGS includes two phases: a pre-stable period and a stable one. The first one comes back to geocasting the message to the specific region. Whenever a vehicle detects such critical event it immediately broadcasts it and keeps rebroadcasting. This process comes to an end as far it receives the same message from a rely vehicle on the opposite side.The most distant vehicle is selected for the relaying mission based on a stable period of time. The ultimate goal of this stable period is to keep alive the message within the geocast region whenever it is still woth of pertinence for this region.

In Abiding Geocast, the information is only disseminated to the geocast area for a specific duration of time in order to keep alive all messages and disseminate them to all vehicles [11]. Hence, three basic approaches are proposed, server-based, neighbors approach and neighbor's election-based approach: Neighbor's approaches are the most used in abiding geocast. They use both unicasting and single-hop broadcasting for data dissemination. The Unicasting is used to transmit the new message to the specified location utilizing infrastructures while broadcasting is used to disseminate the message within the specified location. In Yu et al. [11], the opposite side vehicles are exploited to make an optimized version of [12] safety abiding geocast protocol. Indeed, a dynamic adjustment of waiting time after the next broadcast and the selection of the best opposite lanes vehicles to rebroadcast the message in such critical dangerous event can deal with the broadcast storm problem [13] and avoid unnecessary broadcast.

In the above mentioned works, forms of ZORs, their origins and positioning techniques still arbitrary and are chosen according to scenarios and motivation needs of the authors. For the sake of guaranteeing a high geocast precision by sending message only to vehicles interested by the disseminated event, in this paper, we delegate the assignment of geographic areas of ZORs to a competent authority (e.g., road safety services, the police headquarters, etc). Indeed, in the protocol that we introduce in the remainder, the map is split into a set of regions. Then, for each event that arises in a given region, we associate a set of regions composing its zone of relevance. Hence, a centralized database is dedicated to store the result of map decomposition and regions of interest association.

Furthermore, geocasted message should remain in the ZOR for a desired delay, such that arriving vehicles could be informed. Only few approaches have addressed this issue by rebroadcasting the event during a certain delay, which 
significantly increases network overload. To tackle this issue, in our protocol, whenever a vehicle enters in new region, it can retrieve pertinent events received by other vehicles in the current region. By doing so, pertinent events are kept alive inside the region, without the need for rebroadcasting event messages like do the existing geocast approaches.

\section{Protocol}

In the following, we start by defining the main concepts that will be of use for the remainder. Thus, we suppose that a "Road" stands for a path that links tow cross streets, while a "Region" stands for a set of connected roads.

Definition 1: Zone of Relevance (ZOR) : a set of regions where a given event is pertinent for vehicles in those regions. It is worth of cite that a database is dedicated to store the result of map decomposition and regions of interest association. Figure 1 illustrates an example of a split map, where each $R_{i}$ shows a region in the map. Assume that a given event $E$ arose in region $R_{6}$, then the zone of relevance of $E$ is the set of regions surrounding $R_{6}$, i.e., $Z O R=\left\{R_{1}, R_{2}, R_{3}, R_{5}, R_{6}, R_{7}, R_{9}, R_{10}, R_{11}\right\}$. Moreover, the database stores the log entries of received or detected events.

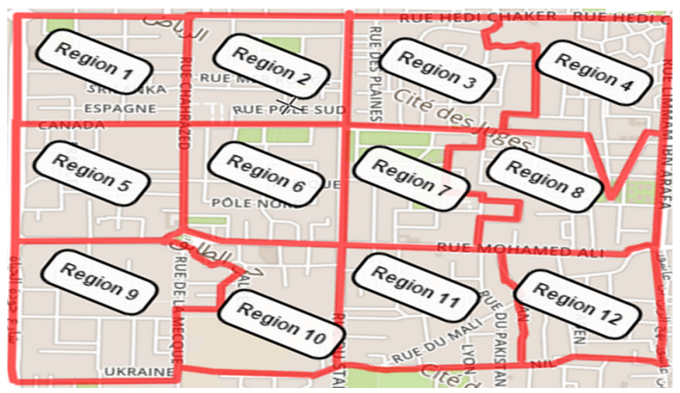

Fig. 1. Example of a split map

In the remainder, we suppose that vehicles are able to determine their position on the road using, e.g., the global positioning system (GPS). We also require that vehicles are able to connect only once to the internet to update their databases by connecting to the database server. In addition, vehicles are equipped with wireless standard for vehicular communication IEEE 802.11p [14] technologies such as Dedicated Short Range Communications (DSRC). Thus, they are able to communicate through an Inter-Vehicle Communication (IVC) manner to enable advanced ITS (Intelligent Transportation System) services.

\section{A. General architecture at a glance}

Figure 2 depicts the DPMS general architecture including the following components:

- database connector: allows to vehicles to connect only once to the centralized database via internet, in order to get a local copy of the database that contains a split offline map of the current city.

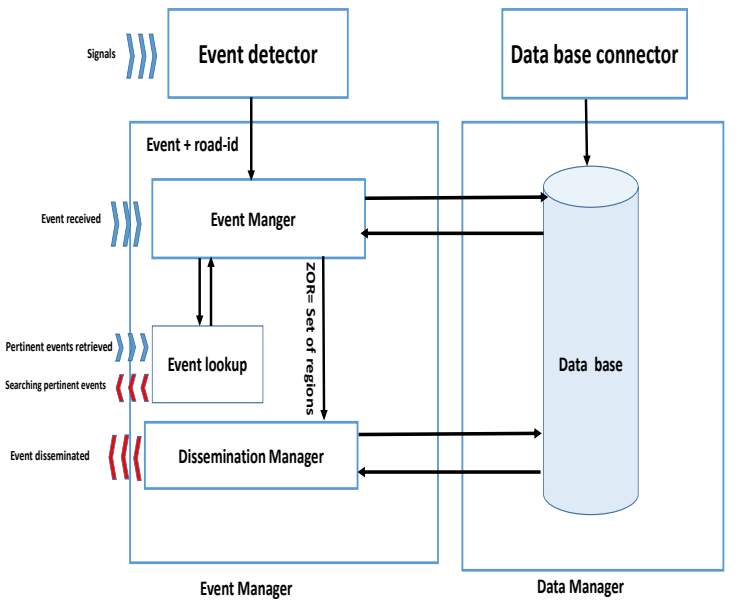

Fig. 2. General DPMS architecture

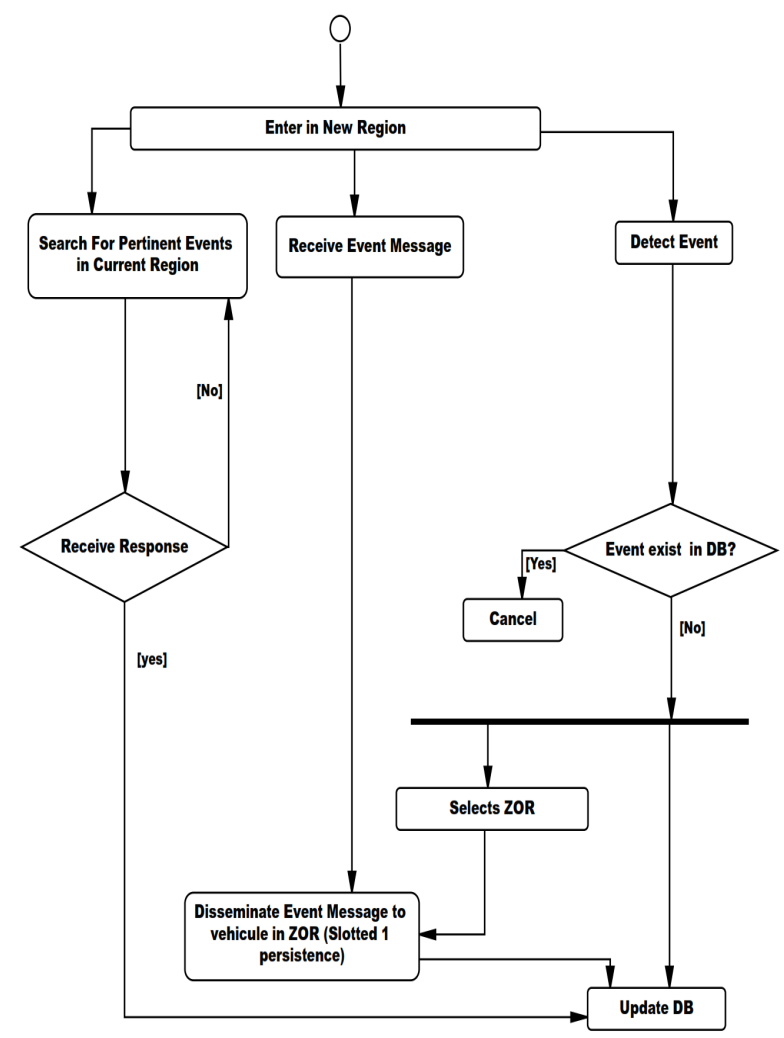

Fig. 3. Protocol Activity Diagram

- Event detector: receives the sensors' signals, detects the events then determines the event-road-id based on the GPS.

- Event lookup: allows vehicle to search for pertinent events received by neighboring vehicles within current region.

- Event manager: determines the event region and its ZOR (i.e., a set of regions) from the database, upon receiving a given event and its road-id from the event detector. There- 
after, it sends the set of regions composing the ZOR to the dissemination manager component. Moreover, event manager invokes the event lookup component whenever the vehicle enters within a new region, then it updates the database whenever a pertinent event occurs.

- Dissemination manager: disseminates events to vehicles within the ZOR using a moderated slotted-1 persistence technique. Indeed, this suppression technique avoids the broadcast storm problem and guarantees a high reachability. In [13], the authors prove that slotted-1 persistence provides 100 percent reachability in a well-connected network and up to approximately 70 percent reduction in broadcast redundancy and packet loss ratio on a well connected vehicular network.

\section{B. Message structure}

The message header structure is therefore defined by the following values: [Sender ID, message ID, time slot, Sender Region, Zone Of Relevance, event type, event time]. The size of the message is less than 2321 Bytes, the maximum allowed size of the message by $802.11 \mathrm{p}$ standard.

\section{Protocol diagram}

In the DPMS protocol, each vehicle gets a local copy of the database that contains a split map of the current city. Then, it has the following situations:

1) Detecting event: Upon detecting a given event $E$, the event detector component sends the event type and its road-id to the event manager. The latter runs this operation to determine the zone of relevance of $E$. Indeed, if $E$ exists in the database (DB) (i.e., $E$ has been already seen), then it will be ignored. Otherwise, the event manager inserts $E$ in the DB. Thereafter, it accesses to the $\mathrm{DB}$, in order to select the regions, where the message should be disseminated, constituting so ZOR of $E$. Finally, it sends the event $E$ and the list of regions of its $\mathrm{ZOR}$ to the dissemination manager component.

2) Searching for pertinent events: Whenever a vehicle enters in a new region, the event lookup component runs this operation in order to retrieve pertinent events received by vehicles in the current region. By doing so, we significantly shorten the time of need to deliver the event to interested vehicles. Furthermore, pertinent events are kept alive inside the region, without the need for rebroadcasting event messages like do the existing geocast approaches. Interestingly enough, this operation of search can effectively reduce the total exchanged message and decreases consequently the network traffic load.

3) Receiving event message: Upon receiving an event from another vehicle, the event manager runs this operation in order to extract the list of regions composing the ZOR from the event message. Thereafter, it sends the event and the list of regions to the dissemination manager component.
4) Disseminating event message: Upon receiving an event message from the event manager, the dissemination manager runs this operation to disseminate the event. In our case, we adapted the well known Slotted 1Persistence Broadcasting technique to disseminate the event to vehicles within the ZOR. Indeed, if the vehicle receiving the event stands outside the ZOR, then it discards it. Otherwise, the vehicle rebroadcasts the event with probability 1 at the assigned time slot $T_{S_{i j}}$ if it receives the packet for the first time and has not received any duplicates before its assigned time slot. Given the relative distance between vehicles $i$ and $j, D_{i j}$, the average transmission range $R$, and the predetermined number of slots $N s, T_{S_{i j}}$ is computed as follows:

$$
T_{S_{i j}}=S_{i j} \times \tau
$$

where $\tau$ is the estimated one-hop delay, which includes the medium access delay and the propagation delay, and $S_{i j}$ is the assigned slot number, which is computed as follows:

$$
S_{i j}=N_{s}\left(1-\left[\frac{\min \left(D_{i j}, R\right)}{R}\right]\right)
$$

\section{EXPERIMENTATIONS}

In this section, we present the performance evaluation of the DPMS protocol versus DTSG protocol [9] carried out by means of simulations with Veins simulator framework 1 . Veins is an open source simulation framework for InterVehicular Communication (IVC) that combines both road traffic microsimulation model as well as event-based network simulator.

\section{A. Experimental settings}

The road traffic simulation is performed by SUMO [15] and Network simulation is performed by OMNeT++ [16] along with the physical layer modeling toolkit MiXiM ${ }^{2}$, which makes it possible to employ accurate models for radio interference, as well as shadowing by static and moving obstacles.

With those two well-established simulators, Nodes simulated by OMNeT++ 4.1.2 can interact with SUMO to simulate the influence of IVC on road traffic and mobility. In the remainder, we take advantage of these two simulators included in Veins. It provides realistic models for 802.11p DSRC, PHY and MAC layers. The PHY and MAC parameters are defined according to the basic specifications of the 802.11p standard.

Simulation settings have been summarized in Table I. In the MAC layer, we set the transmission power to $40 \mathrm{~mW}$ to achieve approximately $300 \mathrm{~m}$ of interference range and 176 of transmission range. For our simulation, we supposed that the map is already split as could be depicted in Figure 1. Please note that the splitting method is beyond the scope of this paper and stands within the issues that we are planing in

\footnotetext{
${ }^{1}$ http://veins.car2x.org/

${ }^{2}$ http://mixim.sourceforge.net/
} 
the near future to tackle. We have considered three different densities: (i) low density with average of $40 \mathrm{veh} / \mathrm{km}$; (ii) medium density with $60 v e h / \mathrm{km}$; and (iii) high density with $100 \mathrm{veh} / \mathrm{km}$.

\begin{tabular}{|c|c|c|}
\hline Physical Layer & Frequency band & $5.9 \mathrm{GHz}$ \\
\hline & Transmission Power & $40 \mathrm{~mW}$ \\
\hline & Bandwith & $10 \mathrm{MHz}$ \\
\hline Link Layer & Bit Rate & $6 \mathrm{Mbit} / \mathrm{s}$ \\
\hline & CW & 15.1023 \\
\hline & Slot Time & $13 \mathrm{us}$ \\
\hline & SIFS & $32 \mathrm{us}$ \\
\hline Scenarios & DIFS & $58 \mathrm{us}$ \\
\hline & Message Size & $2312 \mathrm{Bytes}$ \\
\hline & Message frequency & $0.5 \mathrm{~Hz}$ \\
\hline & \#Runs & $30 \mathrm{times}$ \\
\hline
\end{tabular}

Simulation SETtingS

\section{B. Evaluation metrics}

The assessment of the performances of our protocol is carried out through two global metrics, namely the Effectiveness and the Efficiency of the Dissemination Protocol, which are detailed in the remainder.

1) Effectiveness: We consider that the dissemination protocol is effective whenever it guarantees a high Reachability, i.e., average delivery ratio, and a high geocast Precision.

Reachability: it assesses the average delivery ratio of dissemination, where the message must reach all intersected vehicles of such an event $e$. The reachability is defined as follows:

$$
\text { Reachability }(e)=\frac{|I I V|}{|I V|}
$$

where $I I V$ stands for the set of interested informed vehicles i.e., only pertinent vehicles for an event $e$, and $I V$ stands for the set of interested vehicles in an event $e$. The average reachability is defined as follows:

$$
\text { AverageReachability }=\frac{\left.\sum \text { Reachability }(e)\right)}{\text { NumberOfEvents }}
$$

Precision: This metric assesses to what extent the protocol is able to only inform pertinent vehicles that are actually interested in a given event $e$. Hence, the challenge would be to obtain higher values of geocasting which is in a snugness connection with the quality of the determination of the geocasting area ${ }^{3}$. The precision metric is defined as follows:

$$
\text { Precision }=\frac{|I I V|}{|A I V|}
$$

where $I I V$ stands for the set of interested informed vehicles i.e., only pertinent vehicles for an event $e$, and $A I V$ stands for the set of all informed vehicles, i.e.,

\footnotetext{
${ }^{3}$ Geocast is a special case of multicast where data should be only disseminated to a special geographic area.
}

pertinent as well as not pertinent vehicles for an event $e$. The average precision is defined as follows:

$$
\text { AveragePrecision }=\frac{\left.\sum \text { Precision }(e)\right)}{\text { NumberOfEvents }}
$$

Figure 4 shows the evolution of the reachability and precision values in different density networks. As expected, using our dissemination protocol, the reachability of information about the event is slightly sharper than the DTSG protocol (i.e., Figure 4 (b)). This is owe to the fact that the ZOR determined by DTGS is greater than that of our protocol. Hence, more vehicles are got in touch, which increases the reachability. Nevertheless, our protocol palliates this drawback owe to a high geocasting precision that only targets interested vehicles and keeps a low overload value. Indeed, Figure 4 (b) shows that DPMS has a high geocasting precision in different network density. It is worth of mention that our protocol increases the precision of DTGS by $100 \%$. Figure 4 also shows that the reachability and precision decrease for both protocols as far as the number of vehicles increases. Hence, the higher the number of vehicles is, the lower the probability to reach the interested vehicles is.

2) Efficiency: We consider that the dissemination protocol is efficient whenever it flags out a minimum network Overload and a minimum network Latency. These metrics are explained in the remainder.

Overload: the overload metric stands for the total number of sent packets. Interestingly enough, the ultimate goal of any dissemination protocol is to avoid the overload problem [13] by looking for minimizing the number of message transmissions in the network. Figure 5 (a) shows the results for a scenario when varying the network density from 1 to $100 \mathrm{~km} / \mathrm{h} / \mathrm{lane}$.

Latency: it refers to the amount of time that is of need to deliver a message to an interested vehicle. The average latency, $A L$, is defined as follows:

$$
A L=\frac{\sum\left(t_{i}-T\right)}{\text { NumberOfInterestedVehicles }}
$$

where $t_{i}$ stands for the arrival time of the event message to a vehicle $i$ and $T$ is the timestamp of the occurrence of the event.

Figure 5 shows that our protocol flags out shorter notification delay (i.e., latency) and minimum network overload than DTSG for all the considered variations of traffic density. Indeed, the overload and the latency of our protocol are smaller by $50 \%$ than DTSG in the worst cases. This can simply be explained by the fact that in our protocol, a vehicle can be informed about pertinent events whenever it contacts an informed vehicle in the current region without having the need for rebroadcasting event messages like do DTSG. Interestingly enough, the experimental results prove that this operation of search effectively reduces the total exchanged message and decreases consequently the network traffic. 

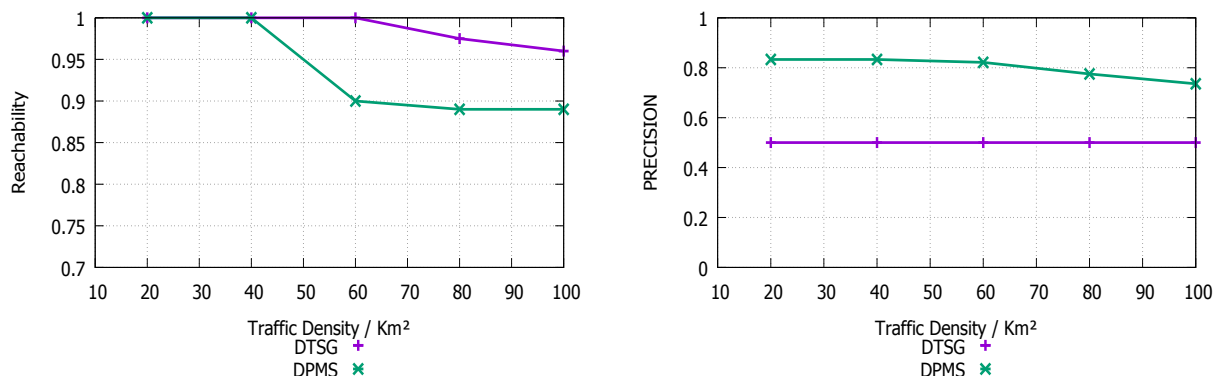

Fig. 4. Variation of the average Reachability/Precision values w.r.t the variation of the number of vehicles
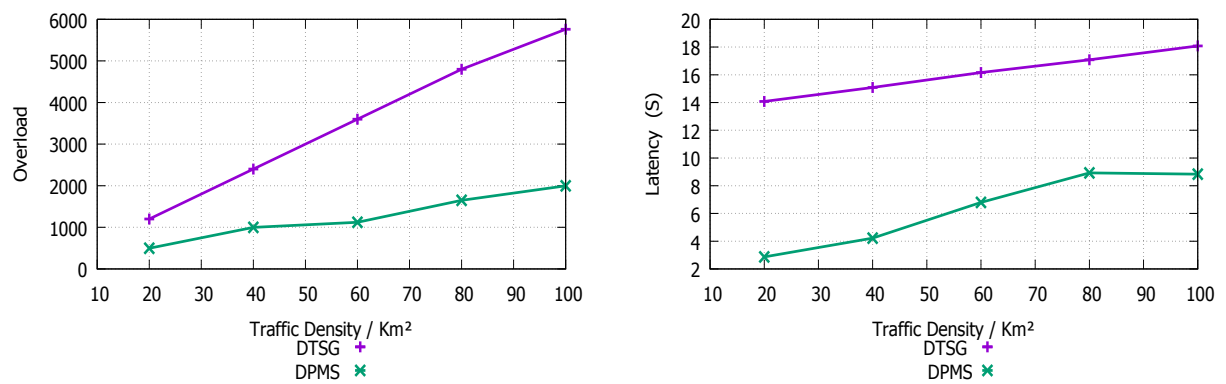

Fig. 5. Variation of the average Overload/Latency values w.r.t the variation of the number of vehicles

\section{CONCLUSIONS AND FUTURE WORK}

In this paper, we introduced, DPMS a MAP-splitting-based dissemination protocol to exchange information about events in a VANET. The main thrust of our protocol stands in an adequate targeting of the zone of relevance of the disseminated messages. Doing so, it allowed us to meet our goals, namely, reaching a high delivery ratio as well as a high geocast precision. Carried out experiments confirmed this fact through the encouraging results obtained by DPMS. In the near future, we plan to tackle the following issues: (i) tackling the smart automatic map splitting through the detection of road's connectivity; (ii) carry out extensive experiments by considering a higher number of cars and (iii) integrate the DPMS protocol within a signal phase and timing information support "green driving" of all vehicles and safe and comfortable crossing of intersections even by blind and visually impaired pedestrians.

\section{REFERENCES}

[1] J. Maurer, T. Fugen, T. Schafer, and W. Wiesbeck, "A new intervehicle communications (ivc) channel model," in Vehicular Technology Conference, 2004. VTC2004-Fall. 2004 IEEE 60th, vol. 1, Sept 2004, pp. 9-13 Vol. 1.

[2] S. Allal and S. Boudjit, "Geocast routing protocols for vanets: Survey and guidelines," in Innovative Mobile and Internet Services in Ubiquitous Computing (IMIS), 2012 Sixth International Conference on, July 2012, pp. 323-328.

[3] B. Bako and M. Weber, "Efficient Information Dissemination in VANETs," in Adv. Veh. Netw. Technol., 2011, p. 20.

[4] B. Alsubaihi and A. Boukerche, "Semantic and self-decision geocast protocol for data dissemination over vanet (sas-gp)," in Wireless Communications and Networking Conference (WCNC), 2015 IEEE, March 2015, pp. 1948-1953.

[5] A. Boukerche and X. Fei, "A coverage-preserving scheme for wireless sensor network with irregular sensing range," Ad Hoc Networks, vol. 5, no. 8, pp. 1303-1316, nov 2007.
[6] S.-H. Lee and Y.-B. Ko, "Geometry-driven scheme for geocast routing in mobile ad hoc networks," in Vehicular Technology Conference, 2006. VTC 2006-Spring. IEEE 63rd, vol. 2, May 2006, pp. 638-642.

[7] L. Zhang, D. Gao, S. Gao, and V. Leung, "Smartgeocast: Dynamic abnormal traffic information dissemination to multiple regions in vanet," in Wireless Communications and Mobile Computing Conference (IWCMC), 2013 9th International, July 2013, pp. 1750-1755.

[8] K. Ibrahim, M. Weigle, and M. Abuelela, "p-ivg: Probabilistic intervehicle geocast for dense vehicular networks," in Vehicular Technology Conference, 2009. VTC Spring 2009. IEEE 69th, April 2009, pp. 1-5.

[9] H. Rahbar, K. Naik, and A. Nayak, "Dtsg: Dynamic time-stable geocast routing in vehicular ad hoc networks," in Ad Hoc Networking Workshop (Med-Hoc-Net), 2010 The 9th IFIP Annual Mediterranean, June 2010, pp. 1-7.

[10] P. Kheawchaoom, S. Kittipiyakul, and K. Na Ayutaya, "idtsg time-stable geocast for post crash notification in vehicular highway networks," in Electrical Engineering/Electronics, Computer, Telecommunications and Information Technology (ECTI-CON), 2012 9th International Conference on, May 2012, pp. 1-4.

[11] Y. Lim, S. Ahn, and K.-H. Cho, "Abiding geocast for commercial ad dissemination in the vehicular ad hoc network," in Consumer Electronics (ICCE), 2011 IEEE International Conference on, Jan 2011, pp. 115-116.

[12] Q. Yu and G. Heijenk, "Abiding geocast for warning message dissemination in vehicular ad hoc networks," in Communications Workshops, 2008. ICC Workshops '08. IEEE International Conference on, May 2008, pp. 400-404.

[13] N. Wisitpongphan, O. Tonguz, J. Parikh, P. Mudalige, F. Bai, and V. Sadekar, "Broadcast storm mitigation techniques in vehicular ad hoc networks," Wireless Communications, IEEE, vol. 14, no. 6, pp. 84-94, December 2007.

[14] J. Kenney, "Dedicated short-range communications (dsrc) standards in the united states," Proceedings of the IEEE, vol. 99, no. 7, pp. 11621182, July 2011.

[15] F. Kaisser, C. Gransart, and M. Berbineau, "Simulations of vanet scenarios with opnet and sumo," in Communication Technologies for Vehicles, ser. Lecture Notes in Computer Science, A. Vinel, R. Mehmood, M. Berbineau, C. Garcia, C.-M. Huang, and N. Chilamkurti, Eds. Springer Berlin Heidelberg, 2012, vol. 7266, pp. 103-112.

[16] A. Varga, "Omnet++," in Modeling and Tools for Network Simulation, K. Wehrle, M. Gne, and J. Gross, Eds. Springer Berlin Heidelberg, 2010, pp. 35-59. 